\title{
4. Beziehungen der Schweiz mit den Entwicklungs- und Transitionsländern
}

\section{Gérard Perroulaz and Christoph Stamm}

\section{(2) OpenEdition \\ 1 Journals}

Electronic version

URL: http://journals.openedition.org/sjep/242

DOI: 10.4000/sjep.242

ISSN: 1663-9677

Publisher

Institut de hautes études internationales et du développement

Printed version

Date of publication: 1 avril 2004

Number of pages: $309-314$

ISSN: 1660-5926

\section{Electronic reference}

Gérard Perroulaz und Christoph Stamm, « 4. Beziehungen der Schweiz mit den Entwicklungs- und

Transitionsländern », Schweizerisches Jahrbuch für Entwicklungspolitik [Online], 23-1 | 2004, Online erschienen am: 03 Mai 2010, abgerufen am 08 September 2020. URL : http://journals.openedition.org/ sjep/242 ; DOI : https://doi.org/10.4000/sjep.242 


\section{Beziehungen der Schweiz mit den Entwicklungs- und Transitionsländern}

\section{1. Übersicht über die Beziehungen der Schweiz mit den Entwicklungsländern und Transitionsländern, 2002}

\begin{tabular}{|c|c|c|c|c|c|c|}
\hline In Tausend Franken & ÖEH/ABÖS ${ }^{1}$ & Priv. Hilfe $\mathrm{e}^{2}$ & $\mathrm{ERG}^{3}$ & $\mathrm{ADI}^{4}$ & Einfuhr & Ausfuhr \\
\hline $\begin{array}{l}\text { Finanzflüsse in } \\
\text { die Entwicklungsländer }\end{array}$ & 1195180 & 298162 & -206674 & 1907000 & 8568242 & 15660340 \\
\hline $\begin{array}{l}\text { Am wenigsten entw. } \\
\text { Länder (LDC) }\end{array}$ & 295674 & 118019 & - & 11400 & 149634 & 530099 \\
\hline $\begin{array}{l}\text { Einkommensschwache } \\
\text { (ohne LDC) }\end{array}$ & 192900 & 59429 & -68020 & -69400 & 3618811 & 3673237 \\
\hline Länd. mit mittl. Einkommen & 263738 & 88916 & -138654 & 561500 & 4610341 & 11133990 \\
\hline Länd. mit höherem Einkommen & 703 & 5 & - & 1379800 & 189456 & 323014 \\
\hline $\begin{array}{l}\text { Regionalprojekte } \\
\text { und nicht aufgegliedert }\end{array}$ & 442165 & 31793 & - & 23700 & - & - \\
\hline $\begin{array}{l}\text { Finanzflüsse in } \\
\text { die Transitionsländer }\end{array}$ & 91839 & 15681 & -26839 & 2054200 & 7796690 & 15390127 \\
\hline FEL & 1034 & 3234 & -13499 & 1725000 & 3750244 & 10634579 \\
\hline OST/NUS & 90805 & 12447 & -13340 & 329200 & 4046446 & 4755548 \\
\hline $\begin{array}{l}\text { Regionalprojekte } \\
\text { und nicht aufgegliedert }\end{array}$ & - & - & - & - & - & - \\
\hline \multicolumn{7}{|l|}{ In Prozent } \\
\hline \multicolumn{7}{|c|}{ Finanzflüsse in die Entwicklungsländer } \\
\hline Am wenigsten entw. Länder (LDC) & 24,7 & 39,6 & n.a. & 0,6 & 1,7 & 3,4 \\
\hline $\begin{array}{l}\text { Einkommensschwache Länder } \\
\text { (ohne LDC) }\end{array}$ & 16,1 & 19,9 & n.a. & $-3,6$ & 42,2 & 23,5 \\
\hline Länder mit mittlerem Einkommen & 22,1 & 29,8 & n.a. & 29,4 & 53,8 & 71,1 \\
\hline Länder mit höherem Einkommen & 0,1 & 0,0 & n.a. & 72,4 & 2,2 & 2,1 \\
\hline $\begin{array}{l}\text { Regionalprojekte } \\
\text { und nicht aufgegliedert }\end{array}$ & 37,0 & 10,7 & - & 1,2 & - & - \\
\hline \multicolumn{7}{|c|}{ Finanzflüsse in die Transitionsländer } \\
\hline FEL & 1,1 & 20,6 & n.a. & 84,0 & 48,1 & 69,1 \\
\hline OST/NUS & 98,9 & 79,4 & n.a. & 16,0 & 51,9 & 30,9 \\
\hline Regionalprojekte/nicht aufgegl.-- & - & - & - & - & - & \\
\hline
\end{tabular}

Quellen für die Tabelle 4.1, 4.2, 4.3: Quelle: DEZA, Statistiques 2002, Aide de la Suisse aux pays en développement et aux pays en transition, Bern, 2004; Eidg. Zollverwaltung, Statistik nach Waren und Ländern, Bern, 2003.

Fussnoten für die Tabellen 4.1, 4.2, 4.3. n.a.: nicht anwendbar

1 ÖEH = öffentliche Entwicklungshilfe

ABÖS $=$ Andere Beiträge des öffentlichen Sektors

2 Priv. Hilfe $=$ private Hilfe der NRO

(Aus Eigenmitteln der NRO, ohne öffentliche Beiträge finanzierte Projekte)

3 ERG $=$ Exportrisikogarantie

4 ADI = Ausländische Direktinvestitionsflüsse 


\subsection{Beziehungen mit den Entwicklungsländern, 2002, in Tausend Franken}

\begin{tabular}{|c|c|c|c|c|c|c|}
\hline & ÖEH/ABÖS & Priv. Hilfe ${ }^{2}$ & $\mathrm{ERG}^{3}$ & $\mathrm{ADI}^{4}$ & Einfuhr & Ausfuhr \\
\hline Gesamtbetrag & 1195180,0 & 298162,0 & $-206674,0$ & 1907000,0 & 8568242 & 15660340 \\
\hline Europa & 127109,0 & 18589,0 & $-82900,0$ & 1680100,0 & 679326 & 2364198 \\
\hline $\begin{array}{l}\text { Einkommensschwache } \\
\text { Länder (ohne LDC) }\end{array}$ & 3355,0 & 46,0 & - & - & 1424 & 7717 \\
\hline Moldawien & 3355,0 & 46,0 & - & - & 1424 & 7717 \\
\hline Länd. mit mittl. Einkommen & en 115349,0 & 17721,0 & $-82900,0$ & 299500,0 & 488446 & 2033467 \\
\hline Albanien & 14649,0 & 1804,0 & - & - & 542 & 21668 \\
\hline Bosnien-Herzegowina & 24555,0 & 3597,0 & - & - & 4613 & 35465 \\
\hline Kroatien & 2418,0 & 164,0 & $-6618,0$ & 6700,0 & 42118 & 201911 \\
\hline $\begin{array}{l}\text { Staaten Ex-Jugoslawiens, } \\
\text { nicht spezifiziert }\end{array}$ & us, 19867,0 & 52,0 & - & - & - & 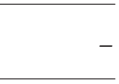 \\
\hline Mazedonien & 9688,0 & 349,0 & - & $-700,0$ & 10495 & 36671 \\
\hline Türkei & 6632,0 & 4151,0 & $-76283,0$ & 280600,0 & 413353 & 1586760 \\
\hline BR Jugoslawien & 37541,0 & 7604,0 & - & 12900,0 & 17326 & 150992 \\
\hline Länd. mit hohem Einkomm & men 703,0 & 5,0 & - & 1379800,0 & 189456 & 323014 \\
\hline Malta & 600,0 & - & - & 20500,0 & 5126 & 61479 \\
\hline Slowenien & 103,0 & 5,0 & - & 1359300,0 & 184329 & 261535 \\
\hline $\begin{array}{l}\text { Nicht aufgegliedert } \\
\text { und Regionalprojekte }\end{array}$ & 7702,0 & 817,0 & - & 800,0 & - & - \\
\hline Afrika & 291699,0 & 100530,0 & $-38931,0$ & $-276800,0$ & 1544148 & 2083380 \\
\hline $\begin{array}{l}\text { Am wenigsten } \\
\text { entwickelte Länder (LDC) }\end{array}$ & 210670,0 & 78740,0 & - & 21800,0 & 51066 & 395982 \\
\hline Angola & 9199,0 & 3463,0 & - & 1200,0 & 116 & 12862 \\
\hline Benin & 8350,0 & 1415,0 & - & . & 165 & 25475 \\
\hline Burkina Faso & 24831,0 & 4568,0 & - & - & 1324 & 1431 \\
\hline Burundi & 3786,0 & 3043,0 & - & - & 1522 & 792 \\
\hline Kap Verde & 1392,0 & - & - & - & 726 & 892 \\
\hline Zentralafrikanische Rep. & p. $\quad 94,0$ & 327,0 & - & - & 112 & 270 \\
\hline Komoren & - & 52,0 & - & - & 157 & 126 \\
\hline DR Kongo & 6870,0 & 5522,0 & - & - & 1637 & 4887 \\
\hline Dschibuti & 165,0 & 0,0 & - & - & 172 & 594 \\
\hline Eritrea & 3337,0 & 1278,0 & - & - & 14 & 1140 \\
\hline Äthiopien & 3301,0 & 5549,0 & - & . & 5024 & 161026 \\
\hline Gambia & 0,0 & 44,0 & - & - & 56 & 4221 \\
\hline Guinea & 1818,0 & 2404,0 & - & 600,0 & 144 & 20408 \\
\hline Äquatorialguinea & 40,0 & 52,0 & - & . & 198 & 564 \\
\hline Guinea-Bissau & 1204,0 & 299,0 & - & - & 0 & 665 \\
\hline Lesotho & 930,0 & 477,0 & - & - & 0 & 74 \\
\hline Liberia & 2601,0 & 1034,0 & - & . & 488 & 15524 \\
\hline Madagaskar & 9364,0 & 2336,0 & - & . & 3795 & 1872 \\
\hline Malawi & 881,0 & 1080,0 & - & . & 8702 & 413 \\
\hline Mali & 12313,0 & 3563,0 & - & . & 1248 & 3303 \\
\hline Mauretanien & 619,0 & 1907,0 & - & . & 68 & 28727 \\
\hline
\end{tabular}

Siehe Fussnoten der vorhergehenden Seite. 


\begin{tabular}{|c|c|c|c|c|c|c|}
\hline & ÖEH/ABÖS ${ }^{1}$ & Priv. Hilfe ${ }^{2}$ & ERG $^{3}$ & $\mathrm{ADI}^{4}$ & Einfuhr & Ausfuhr \\
\hline Mosambik & 33686,0 & 5970,0 & - & 20000,0 & 632 & 5552 \\
\hline Niger & 13921,0 & 1141,0 & - & . & 49 & 20491 \\
\hline Uganda & 891,0 & 4211,0 & - & 7900,0 & 13545 & 4244 \\
\hline Ruanda & 6066,0 & 3172,0 & - & - & 68 & 756 \\
\hline Senegal & 6278,0 & 2101,0 & - & 2700,0 & 3877 & 15531 \\
\hline Sierra Leone & 4632,0 & 397,0 & - & . & 54 & 1473 \\
\hline Somalia & 1079,0 & 1372,0 & - & - & 32 & 25 \\
\hline Sudan & 8060,0 & 9123,0 & - & . & 498 & 27035 \\
\hline Tansania & 30108,0 & 7746,0 & - & $-1600,0$ & 2855 & 14077 \\
\hline Tschad & 12127,0 & 2750,0 & - & - & 0 & 719 \\
\hline Togo & 1035,0 & 1207,0 & - & . & 2087 & 18173 \\
\hline Sambia & 1693,0 & 1136,0 & - & $-9000,0$ & 1410 & 2239 \\
\hline $\begin{array}{l}\text { Einkommensschwache } \\
\text { Länder (ohne LDC) }\end{array}$ & 24665,0 & 12985,0 & $-1190,0$ & $-113500,0$ & 518452 & 258868 \\
\hline Kamerun & 4617,0 & 3367,0 & - & $-27000,0$ & 4177 & 12576 \\
\hline Republik Kongo & 553,0 & 385,0 & - & & 343 & 4733 \\
\hline Elfenbeinküste & 4930,0 & 167,0 & $-708,0$ & $-90300,0$ & 21456 & 26328 \\
\hline Ghana & 11557,0 & 1473,0 & - & $-1900,0$ & 32154 & 17542 \\
\hline Kenia & 934,0 & 4690,0 & $-458,0$ & $-300,0$ & 20863 & 31674 \\
\hline Nigeria & 373,0 & 355,0 & - & 2900,0 & 426287 & 156056 \\
\hline Simbabwe & 1702,0 & 2546,0 & $-24,0$ & 3100,0 & 13172 & 9930 \\
\hline \multicolumn{2}{|c|}{ Länd. mit mittl. Einkommen 22406,0 } & 6206,0 & $-37741,0$ & $-169400,0$ & 974630 & 1428530 \\
\hline Südafrika & 9518,0 & 1795,0 & $-1611,0$ & $-208800,0$ & 620041 & 504340 \\
\hline Algerien & 1147,0 & 712,0 & $-17570,0$ & $-1600,0$ & 194960 & 139167 \\
\hline Botswana & - & 48,0 & - & . & 124 & 3085 \\
\hline Ägypten & 6652,0 & 797,0 & $-7991,0$ & 47500,0 & 20492 & 470749 \\
\hline Gabun & 0,0 & 859,0 & - & 2600,0 & 164 & 7166 \\
\hline Marokko & 1816,0 & 999,0 & $-7600,0$ & 23300,0 & 82822 & 140848 \\
\hline Mauritius & - & 16,0 & $-394,0$ & $-29900,0$ & 30323 & 41337 \\
\hline Namibia & 740,0 & 404,0 & - & . & 2758 & 2531 \\
\hline Seychellen & $-3,0$ & - & - & .. & 29 & 1344 \\
\hline Swasiland & - & 199,0 & - & - & 101 & 1448 \\
\hline Tunesien & 2534,0 & 377,0 & $-2576,0$ & $-2500,0$ & 22808 & 116209 \\
\hline $\begin{array}{l}\text { Nicht aufgegliedert } \\
\text { und Regionalprojekte }\end{array}$ & 33958,0 & 2600,0 & - & $-15700,0$ & - & - \\
\hline Amerika & 126393,0 & 62496,0 & $-190689,0$ & 318700,0 & 1602492 & 3530089 \\
\hline $\begin{array}{l}\text { Am wenigsten entw. } \\
\text { Länder (LDC) }\end{array}$ & 4162,0 & 3547,0 & - & - & 1709 & 1786 \\
\hline Haiti & 4162,0 & 3547,0 & - & - & 1709 & 1786 \\
\hline $\begin{array}{l}\text { Einkommensschwache } \\
\text { Länder (ohne LDC) }\end{array}$ & 12392,0 & 9363,0 & - & $-22500,0$ & 15836 & 37092 \\
\hline Honduras & 2758,0 & 3925,0 & - & $-23100,0$ & 12017 & 29445 \\
\hline Nicaragua & 9635,0 & 5438,0 & - & 600,0 & 3819 & 7647 \\
\hline Länd. mit mittl. Einkomm & nen 83495,0 & 49422,0 & $-190689,0$ & 321700,0 & 1584947 & 3491211 \\
\hline Argentinien & 186,0 & 397,0 & $-3935,0$ & $-462900,0$ & 55424 & 176469 \\
\hline
\end{tabular}




\begin{tabular}{|c|c|c|c|c|c|c|}
\hline & ÖEH/ABÖS ${ }^{1}$ & Priv. Hilfe ${ }^{2}$ & $\mathrm{ERG}^{3}$ & $\mathrm{ADI}^{4}$ & Einfuhr & Ausfuhr \\
\hline Barbados & - & - & - & 13400,0 & 2716 & 3224 \\
\hline Belize & 71,0 & 122,0 & - & - & 76 & 1317 \\
\hline Bolivien & 20807,0 & 8848,0 & $-1193,0$ & 6800,0 & 1076 & 8310 \\
\hline Brasilien & 4348,0 & 9034,0 & $-39477,0$ & $-496700,0$ & 706507 & 1103914 \\
\hline Chile & 1630,0 & 819,0 & $-4091,0$ & 1100,0 & 57642 & 142199 \\
\hline Kolumbien & 12657,0 & 5594,0 & $-864,0$ & $-110500,0$ & 296482 & 180413 \\
\hline Costa Rica & 2318,0 & 596,0 & - & 16800,0 & 87352 & 64323 \\
\hline Kuba & 2311,0 & 468,0 & - & & 30189 & 5901 \\
\hline Dominikanische Repul & bblik 1055,0 & 1592,0 & $-1197,0$ & $-16100,0$ & 19111 & 25473 \\
\hline Dominica & 18,0 & - & - & - & 65 & 960 \\
\hline El Salvador & 6383,0 & 5043,0 & 1273,0 & $-3200,0$ & 7550 & 17844 \\
\hline Ekuador & 9843,0 & 2537,0 & - & 42400,0 & 41525 & 53724 \\
\hline Guatemala & 2674,0 & 5311,0 & $-3538,0$ & 18500,0 & 15248 & 28224 \\
\hline Jamaika & - & 18,0 & - & & 1800 & 18290 \\
\hline Mexiko & 424,0 & 2511,0 & $-153901,0$ & 211900,0 & 184345 & 990062 \\
\hline Montserrat & 38,0 & - & - & - & 3 & 242 \\
\hline Panama & 15,0 & 45,0 & $-365,0$ & 1205100,0 & 43974 & 274732 \\
\hline Paraguay & 1223,0 & 725,0 & $-96,0$ & $-9000,0$ & 2716 & 12760 \\
\hline Peru & 16983,0 & 5132,0 & $-496,0$ & $-11800,0$ & 14579 & 67947 \\
\hline Trinidad und Tobago & - & - & 10716,0 & & 783 & 28776 \\
\hline Uruguay & 423,0 & 96,0 & - & 155000,0 & 8661 & 78884 \\
\hline Venezuela & 89,0 & 537,0 & 6476,0 & $-239100,0$ & 5257 & 199747 \\
\hline $\begin{array}{l}\text { Nicht aufgegliedert } \\
\text { und Regionalprojekte }\end{array}$ & 26343,0 & 164,0 & - & 19500,0 & - & $2=$ \\
\hline Asien & 298459,0 & 90900,0 & 105847,0 & 181100,0 & 4740952 & 7676959 \\
\hline $\begin{array}{l}\text { Am wenigsten entw. } \\
\text { Länder (LDC) }\end{array}$ & 80842,0 & 35725,0 & - & $-10400,0$ & 96772 & 131533 \\
\hline Afghanistan & 20704,0 & 7566,0 & - & & 318 & 1957 \\
\hline Bangladesch & 21160,0 & 3568,0 & - & 200,0 & 64816 & 67949 \\
\hline Bhutan & 6983,0 & 741,0 & - & - & 1 & 516 \\
\hline Kambodscha & 5026,0 & 20214,0 & - & $-8200,0$ & 11907 & 4131 \\
\hline Laos & 3130,0 & 860,0 & - & & 2615 & 1081 \\
\hline Myanmar (Burma) & 1414,0 & 972,0 & - & $-2400,0$ & 10928 & 8122 \\
\hline Nepal & 22425,0 & 1803,0 & - & . & 5665 & 4085 \\
\hline Jemen & 0,0 & 0,0 & - & . & 506 & 41566 \\
\hline $\begin{array}{l}\text { Einkommensschwache } \\
\text { Länder (ohne LDC) }\end{array}$ & 152488,0 & 37036,0 & $-66830,0$ & 66600,0 & 3083099 & 3369560 \\
\hline Armenien & 3583,0 & 1565,0 & - & - & 1383 & 13859 \\
\hline Aserbaidschan & 8802,0 & - & $-1153,0$ & 6400,0 & 540 & 24298 \\
\hline China & 16646,0 & 1206,0 & 3330,0 & $-27900,0$ & 2206905 & 2046492 \\
\hline Nordkorea & 5339,0 & 1453,0 & - & s. & 1397 & 20497 \\
\hline Indien & 41760,0 & 23609,0 & $-17729,0$ & 78600,0 & 516058 & 641257 \\
\hline Indonesien & 9866,0 & 1231,0 & $-48733,0$ & 35000,0 & 168122 & 290718 \\
\hline Kirgistan & 21551,0 & 831,0 & - & - & 135 & 2766 \\
\hline Mongolei & 1991,0 & 551,0 & - & . & 663 & 4363 \\
\hline
\end{tabular}




\begin{tabular}{|c|c|c|c|c|c|c|}
\hline & ÖEH/ABÖS ${ }^{1}$ & Priv. Hilfe $\mathrm{e}^{2}$ & $\mathbf{E R G}^{3}$ & $\mathrm{ADI}^{4}$ & Einfuhr & Ausfuhr \\
\hline Pakistan & 15371,0 & 1075,0 & $-2422,0$ & 17700,0 & 40214 & 203104 \\
\hline Tadschikistan & 8143,0 & 264,0 & - & . & 142 & 1544 \\
\hline Osttimor & 125,0 & 21,0 & - & - & 99 & 152 \\
\hline Turkmenistan & 11,0 & - & - & . & 376 & 3397 \\
\hline Vietnam & 19298,0 & 5230,0 & $-122,0$ & $-43200,0$ & 146903 & 112008 \\
\hline Länd. mit mittl. Einkommen & en 42096,0 & 15136,0 & 172676,0 & 109700,0 & 1561081 & 4175866 \\
\hline Saudi-Arabien & - & 0,0 & $-411,0$ & 6800,0 & 229437 & 1175999 \\
\hline Bahrein & - & - & 181836,0 & 9300,0 & 9244 & 193557 \\
\hline Georgien & 7015,0 & 554,0 & - & . & 162 & 5104 \\
\hline Irak & 3874,0 & 744,0 & - & - & 0 & 123850 \\
\hline Iran & 169,0 & 0,0 & 267,0 & $-21900,0$ & 158806 & 481312 \\
\hline Jordanien & 3240,0 & 122,0 & 718,0 & $-1600,0$ & 389 & 100112 \\
\hline Kasachstan & 104,0 & 1738,0 & - & $-29100,0$ & 58698 & 76702 \\
\hline Libanon & 1022,0 & 1331,0 & 6285,0 & 16600,0 & 151652 & 209469 \\
\hline Malaysia & 55,0 & 319,0 & - & $-33900,0$ & 198447 & 445561 \\
\hline Usbekistan & 5339,0 & - & - & 19000,0 & 2951 & 36661 \\
\hline Philippinen & 1612,0 & 6986,0 & $-8557,0$ & $-20600,0$ & 114505 & 236422 \\
\hline Sri Lanka & 4746,0 & 1235,0 & - & $-6100,0$ & 31130 & 54302 \\
\hline Syrien & 151,0 & 4,0 & - & . & 1173 & 155719 \\
\hline Thailand & 1985,0 & 574,0 & $-7464,0$ & 171200,0 & 601509 & 802823 \\
\hline $\begin{array}{l}\text { Gebiete unter palästi- } \\
\text { nensischer Verwaltung }\end{array}$ & 12785,0 & 1529,0 & - & - & 363 & 3279 \\
\hline $\begin{array}{l}\text { Nicht aufgegliedert } \\
\text { und Regionalprojekte }\end{array}$ & 23034,0 & 3004,0 & - & 15200,0 & - & - \\
\hline Ozeanien & 392,0 & 439,0 & - & 3900,0 & 1324 & 5714 \\
\hline $\begin{array}{l}\text { Am wenigsten entw. } \\
\text { Länder (LDC) }\end{array}$ & - & 8,0 & - & - & 87 & 798 \\
\hline Samoa & - & 8,0 & - & - & 9 & 551 \\
\hline Vanuatu & - & - & - & - & 6 & 231 \\
\hline Länd. mit mittl. Einkommer & 392,0 & 431,0 & - & - & 1237 & 4916 \\
\hline Mikronesien & 60,0 & 0,0 & - & - & 0 & 77 \\
\hline Papua-Neuguinea & 332,0 & 431,0 & - & - & 28 & 745 \\
\hline $\begin{array}{l}\text { Nicht aufgegliedert } \\
\text { und Regionalprojekte }\end{array}$ & - & - & - & 3900,0 & - & - \\
\hline $\begin{array}{l}\text { Nicht nach Kontinenten } \\
\text { aufgegliedert }\end{array}$ & 351127,0 & 25208,0 & - & - & - & - \\
\hline
\end{tabular}




\subsection{Beziehungen mit den Transitionsländern (Hilfe für den Osten und die fortgeschritteneren Entwicklungsländer, 2002, in Tausend Franken}

\begin{tabular}{|c|c|c|c|c|c|c|}
\hline & ÖEH/ABÖS ${ }^{1}$ & Priv. Hilfe ${ }^{2}$ & ERG $^{3}$ & $\mathrm{ADI}^{4}$ & Einfuhr & Ausfuhr \\
\hline Gesamtbetrag & 91839,0 & 15681,0 & $-26839,0$ & 2054200,0 & 7796690 & 15390127 \\
\hline $\begin{array}{l}\text { Fortgeschrittenere } \\
\text { Entwicklungsländer }\end{array}$ & 1034,0 & 3234,0 & $-13499,0$ & 1725000,0 & 3750244 & 10634579 \\
\hline Niederländische Antillen & - & - & 2343,0 & 946700,0 & 310 & 30083 \\
\hline Aruba & - & - & - & 617100,0 & 10 & 9915 \\
\hline Bahamas & - & - & - & $-910900,0$ & 67423 & 55906 \\
\hline Bermudas & - & - & - & 788300,0 & 11 & 16658 \\
\hline Cayman-Inseln & - & - & - & $-544300,0$ & 14813 & 19690 \\
\hline Zypern & - & - & - & 55000,0 & 5733 & 92038 \\
\hline Südkorea & 133,0 & 6,0 & $-2995,0$ & $-8900,0$ & 534569 & 1096434 \\
\hline Vereinigte Arabische Emi & mirate - & - & 3146,0 & $-64200,0$ & 139728 & 945273 \\
\hline Gibraltar & - & - & - & 7400,0 & 67610 & 18438 \\
\hline Hongkong, China & - & 230,0 & $-1753,0$ & $-203300,0$ & 824363 & 4479660 \\
\hline Israel & 807,0 & 1224,0 & $-14240,0$ & $-15100,0$ & 462518 & 644115 \\
\hline Kuweit & - & - & - & 600,0 & 4072 & 225877 \\
\hline Libyen & 25,0 & - & - & . & 488 & 15524 \\
\hline Macao & - & - & - & 100,0 & 5648 & 3863 \\
\hline Singapur & 34,0 & - & - & 1049700,0 & 250004 & 1518145 \\
\hline Chin. Taipeh (Taiwan) & 35,0 & 206,0 & - & 4900,0 & 604715 & 1185250 \\
\hline $\begin{array}{l}\text { Fortgeschritt. Entwicklung } \\
\text { länder, nicht aufgegliedert }\end{array}$ & ggs- & - & - & 1900,0 & - & - \\
\hline $\begin{array}{l}\text { Mittel- und osteuropäisch } \\
\text { Länder, Neue } \\
\text { unabhängige Staaten }\end{array}$ & $\begin{array}{l}\text { che } \\
90805,0\end{array}$ & 12447,0 & $-13340,0$ & 329200,0 & 4046446 & 4755548 \\
\hline Weissrussland & 2855,0 & 43,0 & - & & 2575 & 25685 \\
\hline Bulgarien & 8451,0 & 681,0 & - & 124200,0 & 68791 & 152835 \\
\hline Estland & 88,0 & - & - & 900,0 & 35270 & 36892 \\
\hline Ungarn & 175,0 & 544,0 & - & 23600,0 & 644063 & 716021 \\
\hline Lettland & 243,0 & 77,0 & $-1319,0$ & 45800,0 & 10961 & 101257 \\
\hline Litauen & 173,0 & 68,0 & $-4689,0$ & 30400,0 & 24868 & 75194 \\
\hline Polen & 346,0 & 1293,0 & $-9176,0$ & $-175200,0$ & 471742 & 1140682 \\
\hline Rumänien & 8202,0 & 4479,0 & 7682,0 & 2200,0 & 106908 & 244253 \\
\hline Russland & 26126,0 & 1985,0 & - & 128500,0 & 1527145 & 882215 \\
\hline Slowakische Republik & 4179,0 & 335,0 & $-5838,0$ & 52300,0 & 279281 & 260425 \\
\hline Tschechische Republik & 10760,0 & 1261,0 & - & 56800,0 & 842151 & 945345 \\
\hline Ukraine & 6546,0 & 707,0 & - & 39600,0 & 32690 & 174742 \\
\hline $\begin{array}{l}\text { MOEL/NUS } \\
\text { nicht aufgegliedert }\end{array}$ & 22660,0 & 974,0 & - & 100,0 & - & - \\
\hline $\begin{array}{l}\text { Hilfe für die Transitions- } \\
\text { länder, nicht aufgegliedert }\end{array}$ & $\mathrm{rt}$ & - & - & - & - & - \\
\hline
\end{tabular}

